\title{
Cytoplasmic ends of tetraspanin 7 harbour epitopes recognised by autoantibodies in type 1 diabetes
}

\author{
Anne Eugster ${ }^{1}$ - Gloria Kraus ${ }^{1} \cdot$ Vicky Lidzba $^{1}$ - Denise Müller ${ }^{1}$ - Manja Jolink ${ }^{2,3}$ - Anette-G. Ziegler ${ }^{2,3,4}$. \\ Ezio Bonifacio ${ }^{1,5}$
}

Received: 20 November 2018 / Revised: 21 December 2018 / Accepted: 15 January 2019 / Published online: 21 February 2019

(C) Springer-Verlag GmbH Germany, part of Springer Nature 2019

\begin{abstract}
Aims/hypothesis The beta cell protein tetraspanin 7 is a target of autoantibodies in individuals with type 1 diabetes. The aim of this study was to identify autoantibody epitope-containing regions and key residues for autoantibody binding.

Methods Autoantibody epitope regions were identified by immunoprecipitation of luciferase-tagged single or multiple tetraspanin 7 domains using tetraspanin 7 antibody-positive sera. Subsequently, amino acids (AAs) relevant for autoantibody binding were identified by single AA mutations.

Results In tetraspanin 7 antibody-positive sera, antibody binding was most frequent to tetraspanin 7 proteins that contained the $\mathrm{NH}_{2}$-terminal cytoplasmic domain 1 (C1; up to $39 \%$ ) or $\mathrm{COOH}$-terminal $\mathrm{C} 3$ (up to $22 \%$ ). Binding to $\mathrm{C} 3$ was more frequent when the domain was expressed along with the flanking transmembrane domain, suggesting that conformation is likely to be important. Binding to external domains was not observed. Single AA mutations of C3 identified residues Y246, E247 and R239 as critical for COOH-terminal binding of 9/10, 10/10 and 8/10 sera tested, respectively. Mutation of cysteines adjacent to the transmembrane domain at either residues C235 or C236 resulted in both decreased (8/178 and 15/178 individuals, respectively; >twofold decrease) and increased (30/178 and 13/178 individuals, respectively; >twofold increase) binding in participant sera vs wild-type protein.
\end{abstract}

Conclusions/interpretation We hypothesise that conformation and, potentially, modification of protein terminal ends of tetraspanin 7 may be important for autoantibody binding in type 1 diabetes.

Keywords Autoantibodies · Epitopes · Glima 38 - Tetraspanin 7 - Type 1 diabetes mellitus

\author{
Abbreviations \\ AA Amino acid \\ C Cytoplasmic domain (1/2/3)
}

Ezio Bonifacio

ezio.bonifacio@tu-dresden.de

1 DFG-Centre for Regenerative Therapies Dresden, Faculty of Medicine Carl Gustav Carus, Technische Universität Dresden, Fetscherstrasse 105, 01307 Dresden, Germany

2 Institute of Diabetes Research, Helmholtz Zentrum München, Munich, Germany

3 Forschergruppe Diabetes, Technische Universität München, Klinikum Rechts der Isar, Munich, Germany

4 Forschergruppe Diabetes e.V. at Helmholtz Zentrum München, Munich, Germany

5 Paul Langerhans Institute Dresden, Helmholtz Centre Munich, Faculty of Medicine Carl Gustav Carus, Technische Universität Dresden, Dresden, Germany
E Extracellular domain (1/2)

RLU Relative light units

SDS Standard deviation score

TM Transmembrane domain (1/2/3/4)

WT Wild-type

\section{Introduction}

Tetraspanin 7 is expressed in pancreatic islet beta cells and is a target of autoantibodies in $35 \%$ of individuals with type 1 diabetes [1, 2]. Similar to GAD65 and islet antigen-2 (IA-2), tetraspanin 7 is also expressed in the central nervous system [1-3]. Tetraspanin 7 is a member of the transmembrane 4 superfamily, with four hydrophobic transmembrane domains (TM1-TM4), multiple cytoplasmic domains (C1-C3) and 


\section{Research in context}

\section{What is already known about this subject?}

- Tetraspanin 7 is a target of autoantibodies in type 1 diabetes

- Tetraspanin 7 has three cytoplasmic domains (C1-C3), and two extracellular domains (E1, E2)

What is the key question?

- Do tetraspanin 7 autoantibodies target regions or epitopes that are susceptible to protein modification?

What are the new findings?

- Major autoantibody binding regions of tetraspanin 7 are contained in its cytoplasmic $\mathrm{NH}_{2}$-terminal (C1) and $\mathrm{COOH}-$ terminal (C3) domains

- Changes of residues within a 15 amino acid region in the $\mathrm{C} 3$ domain affect autoantibody binding, including enhancement of binding in some sera

How might this impact on clinical practice in the foreseeable future?

- We hypothesise that protein conformation changes may enhance antigenicity and that reducing events that favour beta cell protein modifications may help prevent or control islet autoimmunity

two extracellular domains (E1 and E2) [4]. Identifying the domains recognised by autoantibodies may provide an understanding of why tetraspanin 7 is targeted by the immune system and whether the targeting antibodies have functional effects on the pancreatic beta cell. The objective of the study was to identify dominant autoantibody domains and to find evidence for protein change as a mechanism for autoimmunity to tetraspanin 7 . We used tetraspanin 7 protein fragments representing structural domains to map autoepitopes recognised by autoantibodies, and mutational analyses to determine key residues that are necessary for autoantibody binding.

\section{Methods}

Participants Sera were obtained from individuals with newonset type 1 diabetes, and from islet autoantibody-negative participants in the TeenDiab cohort of prospectively followed first-degree relatives of individuals with type 1 diabetes $[5,6]$. In total, 299 serum samples from individuals with new-onset type 1 diabetes (male, $n=167$; median age [range], 10.5 years [1.1-20.0 years]; median time since diagnosis [range], 9 days [1-262 days]) and 218 autoantibody-negative control serum samples (male, $n=100$; median age [range], 12.0 years [7.017.4 years]) were used. Operator-blinded coded samples were tested. The ethical committees of Bavaria or the LudwigMaximilians University approved the studies, which were carried out in accordance with the Declaration of Helsinki, as revised in 2000. Informed, written consent was obtained from participants or parents of participants.

Tetraspanin 7 fragment and mutant proteins The truncated C1 (amino acid [AA] 1-16), C1-TM1 (AA 1-40), C1-TM1E1-TM2 (AA 1-75), C1-TM1-E1 (AA 1-56), TM1-E1-TM2C2 (AA 17-86), E1-TM1-C2 (AA 41-86), C2 (AA 76-86), TM2-C2-TM3-E2 (AA 57-213), C2-TM3-E2 (AA 76-213), TM3-E2-TM4-C3 (AA 87-249), E2-TM4-C3 (AA 113-249), TM4-C3 (AA 214-249) and C3 (AA 235-249) tetraspanin 7 fragments $\mathrm{NH}_{2}$-terminally fused to NanoLuc were constructed by PCR amplification from full-length tetraspanin 7 [7] and cloning into pCMV6-AC-IRES-GFP-Puro (Origene, Rockville, MD, USA) containing NanoLuc. Mutations at the C3 residues of the NanoLuc-TM3-E2-TM4-C3 construct were introduced using QuickChange Site-directed Mutagenesis (Agilent, Santa Clara, CA, USA). To produce NanoLuc-tagged antigen, constructs were expressed in human embryonic kidney (HEK) 293 T cells by polyethylenimine transfection with $2 \mu \mathrm{g}$ of DNA (Polysciences, Warrington, PA, USA). Cell pellets were lysed in $20 \mathrm{mmol} / \mathrm{l}$ Tris- $\mathrm{HCl} \mathrm{pH}$ 8.0, $140 \mathrm{mmol} / \mathrm{l} \mathrm{NaCl}, 1 \mathrm{mmol} / 1$ EDTA, $1 \% \mathrm{wt} / \mathrm{vol}$. Triton X-100, Protease Inhibitor Cocktail (Abcam, Cambridge, UK) and Phosphatase Inhibitor Cocktail Set II (Merck Millipore, Millerica, MC, USA) $\left(100 \mu \mathrm{l}\right.$ per $10^{6}$ cells; $30 \mathrm{~min}$ at $\left.4^{\circ} \mathrm{C}\right)$. After centrifugation $(10 \mathrm{~min}$ at $16,000 \mathrm{~g}$ ), the supernatant was collected and used in immunoassays.

Luciferase immunoprecipitation assay for tetraspanin 7 Serum $(2 \mu \mathrm{l})$ was added to duplicate wells of a 96-well 
microfiltration plate (Merck Millipore, Darmstadt, Germany) containing $23 \mu \mathrm{l}$ of assay buffer (Tris buffered saline $\mathrm{pH} 7.2$ plus $0.1 \% \mathrm{wt} / \mathrm{vol}$. Tween 20 , and $0.05 \% \mathrm{wt} / \mathrm{vol}$. Triton X100) with $5 \times 10^{6}$ relative light units (RLU) of NanoLuc-tagged antigen. Plates were incubated in the dark at room temperature for $2 \mathrm{~h}$. Protein-A Sepharose (GE Healthcare, Freiburg, Germany), $1.5 \mathrm{mg}$ per well pre-swollen in assay buffer plus $0.1 \% \mathrm{wt} / \mathrm{vol}$. BSA-low IgG (Life Technologies, Darmstadt, Germany), was added and plates shaken (300 rev/min) at $4^{\circ} \mathrm{C}$ for $1 \mathrm{~h}$. Wells were washed ten times with $200 \mu \mathrm{l}$ assay buffer. Immunoprecipitated activity was measured after the addition of NanoLuc Glo luciferase substrate (Promega, Fitchburg, WI, USA). Binding to the full-length tetraspanin 7 (wild-type [WT] or mutant C235A or C236A) was expressed as units $/ \mathrm{ml}$, which was determined from a calibration curve prepared from a positive sera pool and negative serum included on every plate. Binding to the fragments was expressed as a standard deviation score (SDS; the RLU in samples minus mean RLU of 20 islet autoantibody- and tetraspanin 7-autoantibody-negative control sera divided by the standard deviation of the negative control sera). Competition of binding was measured by performing the assay with (inhibited) and without (uninhibited) lysate from cells that expressed untagged full-length tetraspanin 7. For alanine scanning, binding to $\mathrm{C} 3$ mutant and WT truncation proteins was expressed as arbitrary units, which were determined from a calibration curve of six calibrators prepared from dilutions of rabbit anti-NanoLuc polyclonal antibody (kind gift from Promega) [8] included on every plate. Binding intensities to mutant proteins were expressed as a percentage relative to binding to WT protein. Antibodies to full-length tetraspanin 7 were measured in the 2016 Islet Autoantibody Standardization Program (IASP) workshop. The sensitivity was $32 \%$ and specificity $98.9 \%$.

Statistical analysis Distributions between groups were compared using Mann-Whitney $U$ test, Fisher's exact test and unpaired $t$ test with equal SD. A two-tailed $p$ value $<0.05$ was considered significant. Heatmaps were created using $\mathrm{R}$ Studio (www.rstudio.com/products/rstudio/download/).

\section{Results}

Antibodies to truncated tetraspanin 7-NanoLuc fusion proteins were measured in 41 tetraspanin 7 antibody-positive serum samples from individuals with type 1 diabetes (median [range], 48.4 units $/ \mathrm{ml}$ [5.3-342.0 units $/ \mathrm{ml}]$ ) and 20 islet autoantibody-negative serum samples [7]. Antibody binding $>3$ SDS was observed in serum from individuals with type 1 diabetes for the $\mathrm{C} 1(n=16 ; 39 \%), \mathrm{C} 1-\mathrm{TM} 1(n=6 ; 15 \%), \mathrm{C} 1-$
TM1-E1 $(n=13,32 \%)$, C1-TM1-E1-TM2 $(n=1 ; 2 \%)$, TM1E1-TM2-C2 $(n=5 ; 12 \%)$, TM3-E2-TM4-C3 $(n=9 ; 22 \%)$, E2-TM4-C3 $(n=2 ; 5 \%)$, TM4-C3 $(n=6 ; 15 \%)$ and C3 $(n=$ $8 ; 20 \%$ ) proteins (Fig. 1a). Heatmap representation indicated that $\mathrm{C} 1$ and TM4-C3 were the most common binding sites (Fig. 1b). Some antibodies that strongly bind to full-length tetraspanin 7 did not bind the truncated proteins (Fig. 1b). Binding to the $\mathrm{C} 1$ and TM3-E2-TM4-C3 truncated NanoLuc fusion proteins were inhibited by untagged full-length tetraspanin 7 (Fig. 1c,d).

TM3-E2-TM4-C3 tetraspanin 7 was chosen to measure antibodies against the $\mathrm{COOH}$ end of tetraspanin 7 in 276 individuals with type 1 diabetes and 200 autoantibody-negative relatives of individuals with type 1 diabetes (Fig. 1e). Binding above the 99th centile of the control group was detected in sera from 56 individuals with type 1 diabetes $(20.2 \%$ [95\% CI $16 \%, 25 \%] ; p<0.0001$; data not shown).

Since binding was observed to both the TM3-E2-TM4-C3 and the TM4-C 3 truncated proteins (Fig. 1a), we chose $\mathrm{C} 3$ as likely to contain autoantibody epitopes. Alanine scanning of this 15 residue domain was performed within the TM3-E2TM4-C3 protein, which showed the highest binding of the C3containing proteins (Fig. 2a). The COOH-terminal end of tetraspanin 7 contained an autoantibody binding hotspot, with alanine mutation of Y246 and E247 abolishing binding in nine and ten sera, respectively, from the ten samples analysed, and mutation of residues A243, Q245, M248 and V249 also reducing the binding of some samples. The R239A mutation abolished binding in eight of the ten samples. Mutations of L237 and S238 had the least effect on binding in the ten sera. There was striking heterogeneity in the binding patterns against the mutants.

Binding changes were observed by mutation of cysteines at positions $235(\mathrm{C} 235 \mathrm{~A})$ and $236(\mathrm{C} 236 \mathrm{~A})$; while binding was abolished in serum 4 and markedly reduced in sera 5 and 6 , a marked increase in binding was observed in sera 7 and 9 (Fig. 1a). Each cysteine residue was mutated in full-length tetraspanin 7 and binding examined in 178 serum samples from individuals with type 1 diabetes and 135 serum from control participants (Fig. 2b). Overall positivity against tetraspanin 7 was similar if WT or mutant proteins were used. However, there was both marked increase and decrease in the binding for a number of samples when either C235A or C236A tetraspanin 7 was used as compared with WT tetraspanin 7 (Fig. 2c,d). Sera from $n=30$ (16.9\%) and $n=$ $13(7.3 \%)$ individuals with type 1 diabetes, respectively, had $>$ twofold increased binding over that seen for WT tetraspanin 7 in the presence of the $\mathrm{C} 235 \mathrm{~A}$ or $\mathrm{C} 236 \mathrm{~A}$ substitution, and $n=8(4.5 \%)$ and $n=15(8.4 \%)$ individuals with type 1 diabetes showed a $>$ twofold reduction in binding to these mutated proteins. 
a

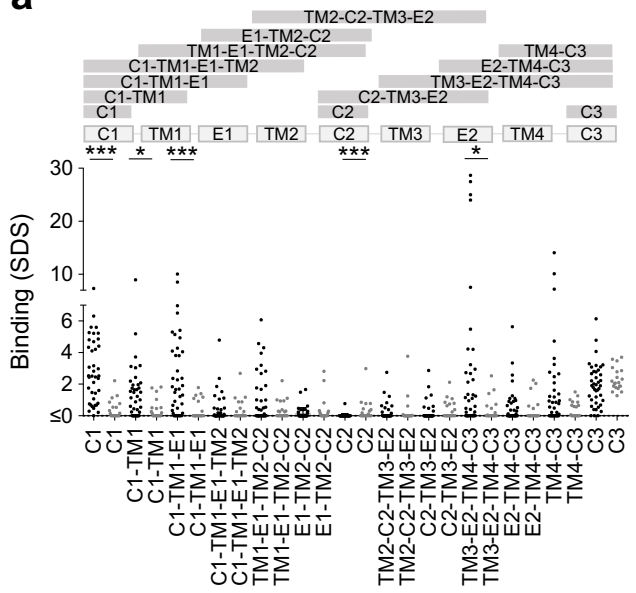

C

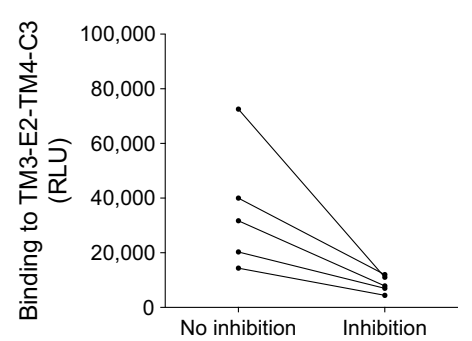

d

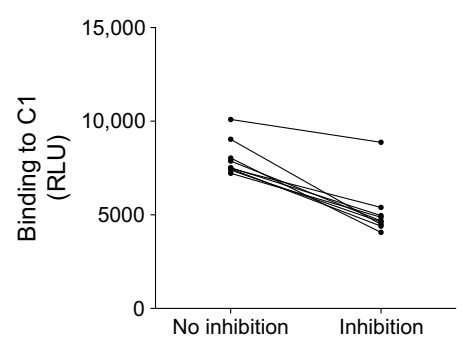

b

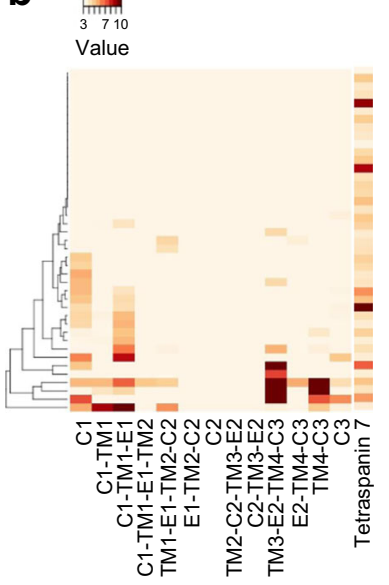

Fig. 1 Autoantibody binding to tetraspanin 7 domains. Binding obtained from luciferase immunoprecipitation assays using tetraspanin 7 domains expressed as in vitro transcribed and translated proteins. (a) Tetraspanin 7 domains and domain combinations used are shown, with binding against each protein in sera from 41 tetraspanin 7 antibody-positive individuals with type 1 diabetes (black circles) and 20 autoantibody-negative control participants (grey circles). Binding is expressed as SDS. (b) Heatmap of binding shown in (a) for sera with SDS $>3$. Sera displaying similar binding properties are grouped through Euclidean clustering. Values shown by colour key correspond to SDS. The value obtained for each serum sample

\section{Discussion}

The cytoplasmic $\mathrm{COOH}$ - and $\mathrm{NH}_{2}$-terminal ends of tetraspanin 7 were identified as targets of autoantibodies in type 1 diabetes. These short ( $<20 \mathrm{AA})$ domains appear to harbour major epitopes of the autoantigen. Autoantibody binding to the $\mathrm{C} 3$ epitopes was increased when the domain was expressed together with its flanking transmembrane region and modifications within the $\mathrm{COOH}$-terminal domain heterogeneously affected autoantibody binding.

The study used samples with moderate-to-high tetraspanin 7 autoantibody titres to identify epitope-containing regions. This was done to reduce the likelihood of missing epitopes due to weak binding, but this selection may have introduced bias into the epitope identification. We did not, for example, identify binding to external domains of tetraspanin 7 , whereas we previously found sera from individuals with type 1 diabetes to contain weak binding to these domains [7]. with full-length tetraspanin 7 is shown in column labelled 'Tetraspanin 7'. (c-d) Competition of binding by non-tagged full-length tetraspanin 7 of autoantibodies from selected individuals showing binding to the TM3E2-TM4-C3 (c) and C1 (d) tetraspanin 7 domain. Data are shown as raw light units expressed as RLU, measured with or without competition (inhibition). (e) Autoantibody binding (raw light units expressed as RLU) to the TM3-E2-TM4-C3 tetraspanin 7 protein in sera from 276 individuals with new-onset type 1 diabetes and 200 autoantibody-negative control participants. $* p<0.05$, $* * * p<0.001$, by Mann-Whitney test in (a) or unpaired $t$ test with equal SD in (e)

The $\mathrm{NH}_{2^{-}}$and $\mathrm{COOH}$-terminal cytoplasmic ends of the protein contain peptidase motifs. The $\mathrm{COOH}$-terminal domain also contains a potential phosphorylation site at T246 and it is of potential interest that alanine mutation of T246 or E247 completely abrogated binding for almost all sera. Of particular interest, changes at cytoplasmic cysteines located at the boundary with the transmembrane 4 region both increased and decreased autoantibody binding, suggesting that some autoantibodies may be directed against a conformationally modified form of the protein. Conformation was important for binding to the $\mathrm{COOH}$-terminal domain, as demonstrated by increased binding when it was expressed together with its flanking transmembrane region. This is consistent with a previous report [9]. We speculate that conformational changes to tetraspanin 7 may occur within islet beta cells, especially during immune, viral or metabolic insults, and that such changes may affect the antigenicity of tetraspanin 7 in individuals with beta cell autoimmunity. 
a

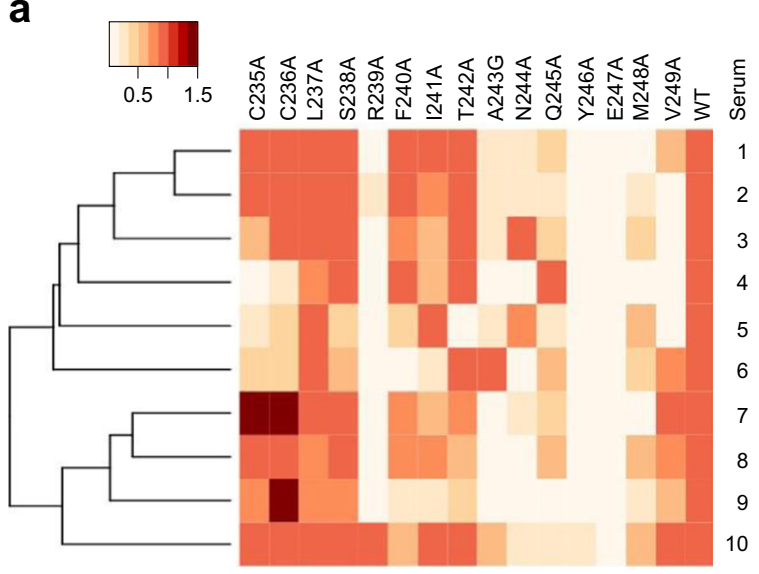

b

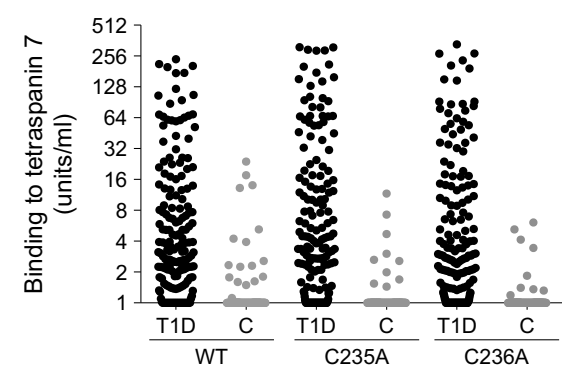

C

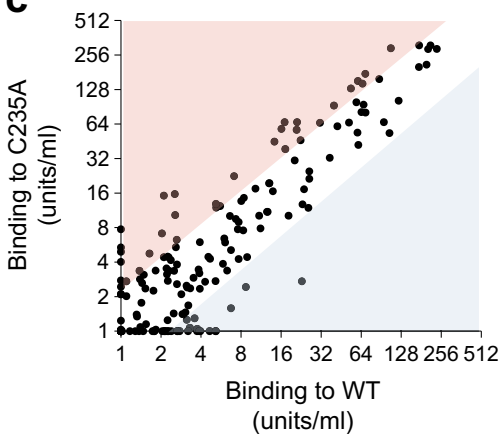

Fig. 2 Alanine scanning to find binding hotspots in C3 of tetraspanin 7. (a) Heatmap showing the binding of ten serum samples from individuals with type 1 diabetes to mutated TM3-E2-TM4-C3 tetraspanin 7 domains expressed as in vitro transcribed and translated proteins. Measured light units (RLU) of mutant proteins are expressed as the percentage of binding of WT protein binding, which is shown in the column labelled 'WT'. The AAs of the C3 domain replaced by alanine are shown above. Sera displaying similar binding properties are grouped through Euclidean clustering. (b) Autoantibody binding (units/ml) to WT full-length tetraspanin 7 , the $\mathrm{C} 235 \mathrm{~A}$ mutated full-length protein and the C236A mutated full-

Data availability The datasets generated and/or analysed during the current study are available from the corresponding author on reasonable request.

Funding This work was funded by the JDRF (2-SRA-2015-13-Q-R) and the DFG FZ 111. Funding agencies had no role in the design of the study or interpretation of the results.

Duality of interest The authors declare that there is no duality of interest associated with the manuscript.

Contribution statement EB and AGZ designed the study, contributed to the conduct of the study, the acquisition, analysis and interpretation of data, and drafted, reviewed and approved the manuscript. AE, GK, VL, $\mathrm{DM}$ and $\mathrm{MJ}$ contributed to the acquisition, analysis and interpretation of data, and drafted, reviewed and approved the manuscript. EB is the guarantor of this work.

Publisher's note Springer Nature remains neutral with regard to jurisdictional claims in published maps and institutional affiliations.

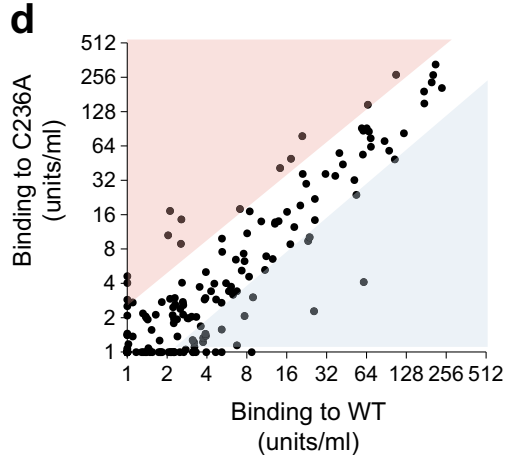

length protein in sera from 178 individuals with type 1 diabetes (T1D; black circles) and 135 control participants (C; grey circles). Binding to tetraspanin 7 (units/ml) is shown on a $\log _{2}$ scale (c-d) Correlation between the binding values as measured for all sera from individuals with type 1 diabetes in (a). Binding to WT ( $x$-axis) vs either the C235A mutant (c) or the C236A mutant (d) ( $y$-axis) is shown on a $\log _{2}$ scale. Red areas, $>$ twofold increased binding to the mutated form vs full-length tetraspanin 7; blue areas, >twofold decreased binding to the mutated form vs fulllength tetraspanin 7

\section{References}

1. McLaughlin KA, Richardson CC, Ravishankar A et al (2016) Identification of tetraspanin-7 as a target of autoantibodies in type 1 diabetes. Diabetes 65(6):1690-1698. https://doi.org/10.2337/ db15-1058

2. Passini N, Larigan JD, Genovese $\mathrm{S}$ et al (1995) The 37/40-kilodalton autoantigen in insulin-dependent diabetes mellitus is the putative tyrosine phosphatase IA-2. Proc Natl Acad Sci U S A 92(20): 9412-9416. https://doi.org/10.1073/pnas.92.20.9412

3. Okada Y, Taniguchi H, Shimada C (1976) High concentration of GABA and high glutamate decarboxylase activity in rat pancreatic islets and human insulinoma. Science 194(4265):620-622. https:// doi.org/10.1126/science. 185693

4. Hemler ME (2005) Tetraspanin functions and associated microdomains. Nat Rev Mol Cell Biol 6(10):801-811. https://doi.org/10. 1038/nrm1736

5. Thümer L, Adler K, Bonifacio E et al (2010) German new onset diabetes in the young incident cohort study: DiMelli study design 
and first-year results. Rev Diabet Stud 7:198-204. https://doi.org/10. 1900/RDS.2010.7.198

6. Ziegler A-G, Meier-Stiegen F, Winkler C, Bonifacio E (2012) Prospective evaluation of risk factors for the development of islet autoimmunity and type 1 diabetes during puberty -TEENDIAB: study design. Pediatr Diabetes 13(5):419-424. https://doi.org/10. 1111/j.1399-5448.2011.00763.x

7. Walther D, Eugster A, Jergens S et al (2016) Tetraspanin 7 autoantibodies in type 1 diabetes. Diabetologia 59(9):1973-1976. https:// doi.org/10.1007/s00125-016-3997-1
8. Hall MP, Unch J, Binkowski BF et al (2012) Engineered luciferase reporter from a deep sea shrimp utilizing a novel imidazopyrazinone substrate. ACS Chem Biol 7(11):1848-1857. https://doi.org/10. $1021 / \mathrm{cb} 3002478$

9. Roll U, Turck CW, Gitelman SE et al (2000) Peptide mapping and characterisation of glycation patterns of the glima 38 antigen recognised by autoantibodies in type I diabetic patients. Diabetologia 43(5):598-608. https://doi.org/10.1007/ s001250051349 